\section{Design of an Enhanced Hybrid Fuzzy P+ID Controller for a Mechanical Manipulator}

\author{
W. Li, X. G. Chang, Jay Farrell, and F. M. Wahl
}

\begin{abstract}
We propose in this paper an enhanced FUZZY P+ID controller to improve control performance in both dynamic transient and steady-state periods for mechanical manipulators under uncertainty. The FUZZY P+ID controller adds only two additional parameters to be tuned relative to the original PID controller. One of these parameters is mainly used to reduce a steady-state error. The other is used to speed up the dynamic response. A simulation study and experimental results for a two-link manipulator with uncertainty demonstrate the superior control performance of the proposed FUZZY P+ID controllers.
\end{abstract}

Index Terms-Fuzzy logic control, PID control hybrid system, robotics manipulator control.

\section{INTRODUCTION}

Industrial manipulators are often equipped with conventional PID controllers due to their simplicity in structure and ease of design. However, when using a PID control, it is difficult to achieve a desired level of control performance, since dynamic equations for mechanical manipulators are tightly coupled and can be highly uncertain (e.g., due to load changes). It is well known that fuzzy logic (FL) controllers based on fuzzy sets [1] are efficient for control of systems with uncertainty [2]-[4]. One of the most widely used design methods for FL controllers is to define membership functions of linguistic variables and to formulate fuzzy rules by control engineers [4]-[6]. However, it is a time consuming process to optimize these fuzzy parameters. Another approach to designing a FL controller is to adapt a rule base and/or membership functions until a desired control performance is achieved [7], [11]. Although provably stable algorithms exist [12]-[17] for special forms of nonlinear systems (e.g., those satisfying matching conditions), stable adaptive approaches for general uncertain nonlinear systems do not yet exist.

Recently, fuzzy-logic and conventional-techniques have been combined to design FL controllers. The hybrid controllers can provide better control performances than PID alone. Furthermore, their stability conditions can be analyzed. For example, Hao [18] was one of the pioneer in the formulation of a hybrid controller and analyzing its control performance. Also, important work in [19] and [20] analyzed the bounded-input/bounded output stability of FUZZY PD and FUZZY PI+FUZZY D control systems by the so-called "small gain theorem." In [21] a new design approach for hybrid FUZZY $\mathrm{P}+\mathrm{ID}$ controller was proposed based on such sufficient stability conditions. The hybrid controller is constructed using an incremental FL controller to replace the proportional term of a conventional PID controller. It is easy to design the FUZZY P+ID controller since it only has one additional parameter to be adjusted relative to its PID

Manuscript received August 6, 1997; revised April 24, 2001. This work was supported by the Alexander von Humboldt Foundation, Bonn, Germany. This paper was recommended by Associate Editor A. Kandel.

W. $\mathrm{Li}$ is with the Department of Computer Science, California State University, Bakersfield, CA 93311 USA.

X. G. Chang is with the National Laboratory for Intelligent Systems and Technology, Department of Computer Science and Technology, Tsinghua University, Beijing, China (e-mail: wli@ee.ucr.edu).

J. Farrell is with the Department of Electrical Engineering, University of California, Riverside, CA 92521 USA

F. M. Wahl is with the Institute for Robotics and Process Control, Technical University of Braunschweig, Braunschweig, Germany.

Publisher Item Identifier S 1083-4419(01)05978-7. counterpart. This approach keeps the simple structure of the PID controller so that it is not necessary to modify any hardware parts of the PID control system for implementation. Its sufficient stability condition [21] shows that the stability behavior remains unchanged when replacing PID control by FUZZY P+ID control. However, this FUZZY P+ID controller suffers from a conflict between reducing the steady-state error and decreasing the rise time when it is used to control mechanical manipulators. In order to solve this problem, we propose an enhanced FUZZY P+ID controller for mechanical manipulators by expanding its rule base from nine to 25 rules, as shown in Fig. 2. Consequently, a desired performance in both transient and steady state periods can be easily achieved. Both the simulation study and experimental results of control on a two-link manipulators with load uncertainty demonstrate the superior control performance of the proposed FUZZY P+ID controllers.

\section{ENHANCED FuZZY P+ID CONTROL SCHEME}

Fig. 1(a) shows a conventional PID controller for a mechanical manipulator. Its control signal for a joint variable, $\theta_{i}(t)$ or $\theta_{i}$, is computed by combining proportional, integral, and derivative terms

$$
\tau_{i}(t)=K_{\mathrm{P} i} e_{i}(t)+K_{\mathrm{I} i} \int e_{i}(t) d t-K_{\mathrm{D} i} \dot{\theta}_{i}(t)
$$

where $K_{\mathrm{P} i}, K_{\mathrm{I} i}$, and $K_{\mathrm{D} i}$ are the controller parameters and $e_{i}(t)=$ $\theta_{r i}(t)-\theta_{i}(t)$. Its discretized and incremental form can be expressed as

$$
\begin{aligned}
\Delta \tau_{i}(k)= & K_{\mathrm{P} i}\left[e_{i}(k)-e_{i}(k-1)\right]+K_{\mathrm{I} i} T e_{i}(k) \\
& -K_{\mathrm{D} i} \frac{\theta_{i}(k)-2 \theta_{i}(k-1)+\theta_{i}(k-2)}{T} .
\end{aligned}
$$

Due to its structural simplicity, the PID controller is widely used in control of industrial manipulators. To improve its control performance, we propose the hybrid FUZZY P+ID controller shown in Fig. 1(b), which is formed by using an incremental FL controller in place of the proportional term. The integral and derivative terms remain the same

$$
\begin{aligned}
\Delta \tau_{i}(k)= & K_{\mathrm{P} i} \Delta u_{i}(k)+K_{\mathrm{I} i} T e_{i}(k) \\
& -K_{\mathrm{D} i} \frac{\theta_{i}(k)-2 \theta_{i}(k-1)+\theta_{i}(k-2)}{T}
\end{aligned}
$$

where $\Delta u_{i}(k)$ is the output of the incremental FL controller. The incremental FL controller has two inputs, $e(k)$ and $\dot{e}(k)$, and an output, $u(k)$. In [21], a FUZZY P controller with nine rules is proposed and its membership functions are given in Fig. 2(a). The division of the operating area $\mathrm{D}$ into 36 cells based on the nine rules is defined by the min function operating on the membership functions. In this paper, the enhanced FUZZY P controller with 25 rules is proposed. Its membership functions (NB, NS, ZO, PS, PB) are defined relative to Fig. 2(b) as follows:

$$
\begin{aligned}
& \mu(x . \mathrm{nb})= \begin{cases}1 & x \leq-\beta \\
\frac{x+\alpha}{\alpha-\beta} & -\beta<x \leq-\alpha\end{cases} \\
& \mu(x . \mathrm{ns})= \begin{cases}\frac{x+\beta}{\beta-\alpha} & -\beta<x \leq-\alpha \\
-\frac{x}{\alpha} & -\alpha<x \leq 0\end{cases} \\
& \mu(x . z o)= \begin{cases}\frac{x+\alpha}{\alpha} & -\alpha<x \leq 0 \\
\frac{\alpha-x}{\alpha} & 0<x \leq \alpha\end{cases}
\end{aligned}
$$




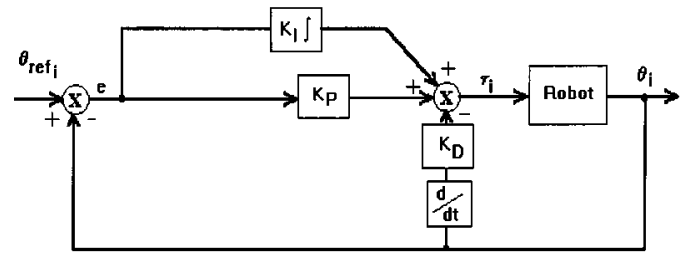

(a)

Fig. 1. Control schemes. (a) PID. (b) FUZZY P+ID.

$$
\begin{aligned}
& \mu(x . \mathrm{ps})= \begin{cases}\frac{x}{\alpha} & 0<x \leq \alpha \\
\frac{x-\beta}{\alpha-\beta} & \alpha<x \leq \beta\end{cases} \\
& \mu(x . \mathrm{pb})=\left\{\begin{array}{ll}
\frac{x-\alpha}{\beta-\alpha} & \alpha<x \leq \beta \\
1 & \beta \leq x
\end{array} .\right.
\end{aligned}
$$

In this study, the variable $x$ is $e(k)$ or $\dot{e}(k)$. Thus, $e(k)$ and $\dot{e}(k)$ can be represented by (e.nb, e.ns, e.zo, e.ps, e.pb) and ( $\dot{e} . \mathrm{nb}, \dot{e} . \mathrm{ns}$, $\dot{e} . z o, \dot{e} . \mathrm{ps}, \dot{e} . \mathrm{pb})$, respectively. For $\Delta u(k)$, we define $o . \mathrm{nb}=-\lambda$, $o . \mathrm{ns}=-\gamma, o . \mathrm{zo}=0, o . \mathrm{ps}=\gamma$ and $o . \mathrm{pb}=\lambda$. For the following discussion we choose $\lambda=\beta$ and $\gamma=\alpha$. The fuzzy rule base of the incremental FL controller is fixed, as shown in Table I, which is used to characterize the relationship between fuzzy inputs and fuzzy outputs. In the rule base shown in Table I, only Zadeh's logical "AND" (i.e., the MIN operator) is required. Since the control actions are described in a fuzzy sense, the "center of mass" defuzzification method [18]-[20] is used to transform fuzzy control actions into crisp outputs as shown in (9) at the bottom of the page.

Due to the MIN-operation each fuzzy region will be represented by five output functions. According to the defined membership functions of (4)-(8), the "min" implementation of the "and" operator, and the fuzzy rule base in Table I, the incremental FL controller is expressed by the hundred functions

$$
\begin{aligned}
\Delta u(k) & =F L C(e(k), \dot{e}(k)) \\
& =\left\{\Delta u^{(1)}(k), \ldots, \Delta u^{(i)}(k), \ldots, \Delta u^{(100)}(k)\right\}
\end{aligned}
$$

that each depend on the same two parameters $\alpha$ and $\beta$. Note that $F L C(e(k), \dot{e}(k))$ is a continuous function of both inputs. Also, since (10) is an antisymmetrical function, we have

$$
\Delta u^{(i)}(k)=-\Delta u^{(100-i+1)}(k) \quad i=1, \ldots, 50 .
$$

As an example, we take $\Delta u^{(24)}(k)$ to explain how $\Delta u^{(i)}(k)$ is computed. In region $24(e(k) \in[-\alpha,-\mathrm{a}], \dot{e}(k) \in[\alpha, \mathrm{b}])$. Therefore, as shown in Fig. 3 and Table I, the following rules are activated:

Rule A) If $e(k)=\mathrm{NS}$ and $\dot{e}(k)=\mathrm{PB}$ then $\Delta u(k)=o . \mathrm{ps}=\gamma$.

Rule B) If $e(k)=\mathrm{NS}$ and $\dot{e}(k)=\mathrm{PS}$ then $\Delta u(k)=o . \mathrm{zo}=0$.

Rule C) If $e(k)=\mathrm{ZO}$ and $\dot{e}(k)=\mathrm{PB}$ then $\Delta u(k)=o \cdot \mathrm{pb}=\lambda$.

Rule D) If $e(k)=\mathrm{ZO}$ and $\dot{e}(k)=\mathrm{PS}$ then $\Delta u(k)=o$.ps $=\gamma$.

For Rule $\mathbf{A}$, we have $0.5 \leq \mu(e . n s) \leq 1$ and $0 \leq \mu(\dot{e}$.pb $)<0.5$, and thus $\{\mu(e . n s)$ AND $\mu(\dot{e} \cdot \mathrm{pb})\}=\min (\mu(e . \mathrm{ns}), \mu(\dot{e} \cdot \mathrm{pb}))=\mu(\dot{e} \cdot \mathrm{pb})$ and $\Delta u(k)=o . p s=\alpha$. For Rule $\mathbf{B}$, we have $0.5 \leq \mu(e . n s) \leq 1$ and $0.5 \leq \mu(\dot{e} . \mathrm{ps}) \leq 1$. Since $\mu(e . n s)$ and $\mu(\dot{e} . \mathrm{ps})$ change

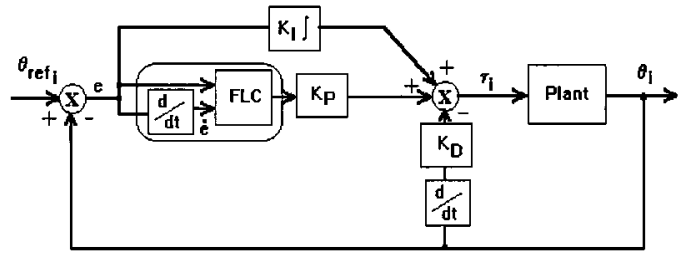

(b)

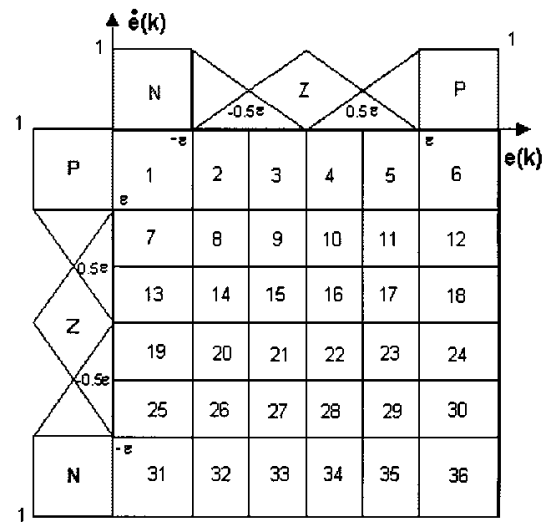

(a)

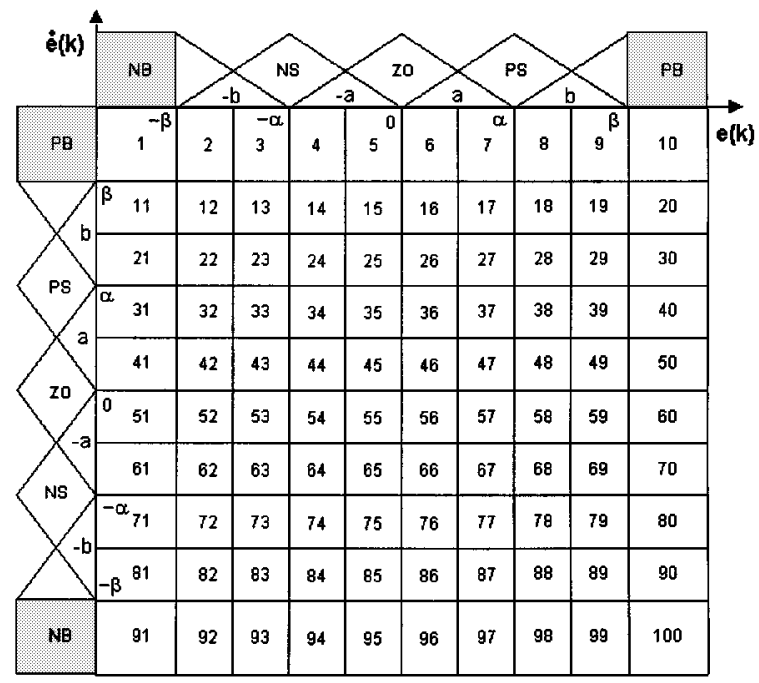

(b)

Fig. 2. Membership functions along each axis are specified along the left and top edges. The combined input regions that result from the "min" implementation of the "and" operation are shown in the interior of the operating region. (a) Controller with nine rules. The "min" operation results in 36 distinct regions. (b) Controller with 25 rules. The "min" operation results in 100 distinct regions.

in the same range $[0.5,1.0]$, the MIN-operation is controlled as follows: If $|-e(k)| \leq|\dot{e}(k)|,\{\mu(e . n s)$ AND $\mu(\dot{e} . \mathrm{ps})\}=\min \{\mu(e . \mathrm{ns}), \mu(\dot{e} . \mathrm{ps})\}=\bar{\mu}(e . \mathrm{ns}) ;$ If $|-e(k)|>|\dot{e}(k)|$, $\{\mu(e . n s)$ AND $\mu(\dot{e} . \mathrm{ps})\}=\min \{\mu(e . n s), \mu(\dot{e} . \mathrm{ps})\}=\mu(\dot{e} . \mathrm{ps})$. The corresponding output value is $\Delta u(k)=o . z o=0$. For Rule $\mathbf{C}$, we 
TABLE I

FUZZY RULE BASE OF THE INCREMENTAL FUZZY LOGIC CONTROLLER

\begin{tabular}{c|c|c|c|c|c}
\hline$\dot{e}$ & NB & NS & ZO & PS & PB \\
\hline PB & ZO & PS & PB & PB & PB \\
\hline PS & NS & ZO & PS & PB & PB \\
\hline ZO & NB & NS & ZO & PS & PB \\
\hline NS & NB & NB & NS & ZO & PS \\
\hline NB & NB & NB & NB & NS & ZO \\
\hline
\end{tabular}
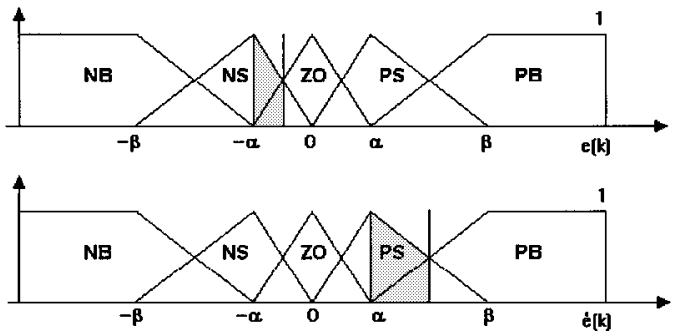

Fig. 3. Control actions in region 8 .

have $0 \leq \mu(e . z o)<0.5$ and $0 \leq \mu(\dot{e} . \mathrm{pb})<0.5$. Since $\mu(e . z o)$ and $\mu(\dot{e} . \mathrm{pb})$ change in the same range $[0,0.5]$, the MIN-operation is controlled as follows: If $|-e(k)| \leq|\dot{e}(k)|,\{\mu(e . z o)$ AND $\mu(\dot{e} \cdot \mathrm{pb})\}=$ $\min \{\mu(e . z o), \mu(\dot{e} . \mathrm{pb})\}=\mu(e . z o) ;$ If $|-e(k)|>|\dot{e}(k)|,\{\mu(e . z o)$ AND $\mu(\dot{e} \cdot \mathrm{pb})\}=\min \{\mu(e . z \mathrm{o}), \mu(\dot{e} \cdot \mathrm{pb})\}=\mu(\dot{e} \cdot \mathrm{pb})$. The corresponding output value is $\Delta u(k)=o . \mathrm{pb}=\beta$. For Rule D, we have $0 \leq \mu(e . z o)<0.5$ and $0.5 \leq \mu(\dot{e}$.ps $) \leq 1$, and thus $\{\mu(e . z o)$ AND $\mu(\dot{e} . \mathrm{ps})\}=\min (\mu(e . z o), \mu(\dot{e} . \mathrm{ps}))=\mu(e . z o)$ and $\Delta u(k)=o . p s=\alpha$. By the "center of mass" formula (8), we have (12), as shown at the bottom of the page, where $a=0.5 \alpha$ and $b=0.5(\alpha+\beta)$. By substituting (4)-(8) and the corresponding output values, $\Delta u^{(24)}(k)$ in the Appendix results. The Appendix lists the equations for $\left\{\Delta u^{(1)}(k), \ldots, \Delta u^{(i)}(k), \ldots, \Delta u^{(50)}(k)\right\}$. In the enhanced hybrid FUZZY P+ID controller, there exist only two additional parameters $\alpha$ and $\beta$ to be tuned. The parameter $\alpha$ mainly affects the steady-state error; while $\beta$ mainly affects the dynamic response.

In order to compare the enhanced FUZZY P+ID controller with the previous nine rule FUZZY P+ID controller, Fig. 4 shows the time response of a nonlinear system controlled as in [21] and controlled as described in the this section. It can be seen that the control performance achieved by the enhanced FUZZY P+ID is better than that

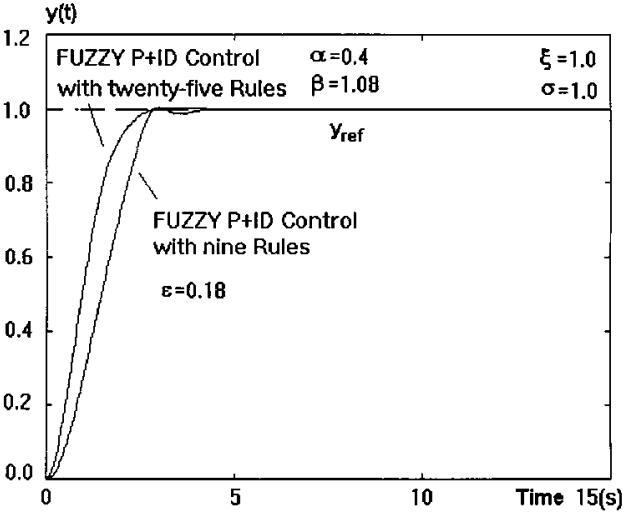

Fig. 4. Comparison of performance of the FUZZY and enhanced FUZZY P+ID controllers.

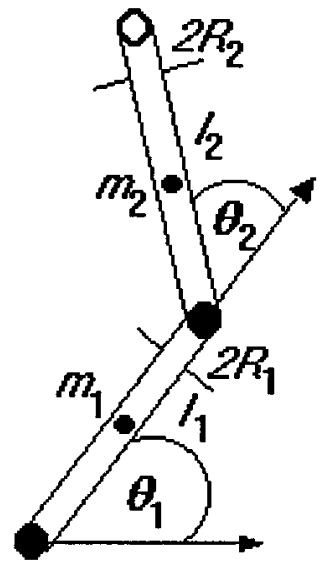

Fig. 5. Two-link mechanical manipulator used for simulation experiments.

achieved by the controller of [21]. It is also much easier to tune the enhanced FUZZY P+ID than its original FUZZY P+ID because the original FUZZY P+ID required a compromise between the dynamic transient response and steady-state error due to the availability of a single tuning parameter. In [22], the sufficient stability conditions for the enhanced FUZZY P+ID controller are discussed based on the "small gain theory" [23], [24].

\section{Simulation Study ON CONTROL OF A Two-LinK MANIPUlator}

In this section, we simulate control performance of the two-link mechanical manipulator, sketched in Fig. 5. The dynamic equations of the manipulator are given in [6]. First, we tune the PID control parameters based on the approaches in [25] and [26] to obtain acceptable control performance. It is very difficult to achieve good control performance from the manipulator by PID control due to the nonlinearity and uncertainty. Table II lists the manipulator data and the controller parameters.

We then design the parameters of the enhanced FUZZY P+ID controller based on its original PID controllers. Based on the stability conditions of the FUZZY P+ID controller in [22], the parameters $K_{\mathrm{P} 1}^{*}$

$$
\begin{aligned}
\Delta u^{(24)}(k)= \begin{cases}\frac{\mu(\dot{e} . \mathrm{pb}) \times o . \mathrm{ps}+\mu(e . \mathrm{ns}) \times o . z o+\mu(\dot{e} . \mathrm{pb}) \times o . \mathrm{pb}+\mu(e . z o) \times o . \mathrm{ps}}{\mu(\dot{e} . \mathrm{pb})+\mu(e . \mathrm{ns})+\mu(\dot{e} \mathrm{pb})+\mu(e . \mathrm{zo})}, & \text { if }|\mu(e . \mathrm{ns})| \leq|\mu(\dot{e} . \mathrm{ps})| \mid \\
\frac{\mu(\dot{e} . \mathrm{pb}) \times o . \mathrm{ps}+\mu(\dot{e} . \mathrm{ps}) \times o . z 0+\mu(e . \mathrm{zo}) \times o . \mathrm{pb}+\mu(e . \mathrm{zo}) \times o . \mathrm{ps}}{\mu(\dot{e} . \mathrm{pb})+\mu(\dot{e} . \mathrm{ps})+\mu(e . \mathrm{zo})+\mu(e . z o)}, & \text { if }|\mu(e . \mathrm{ns})|>|\mu(\dot{e} . \mathrm{ps})|\end{cases} \\
\alpha \leq e(k) \leq-\mathrm{a}, \quad \alpha \leq \dot{e}(k) \leq \mathrm{b}
\end{aligned}
$$


TABLE II

MANIPULATOR DATA AND CONTROLLER PARAMETERS

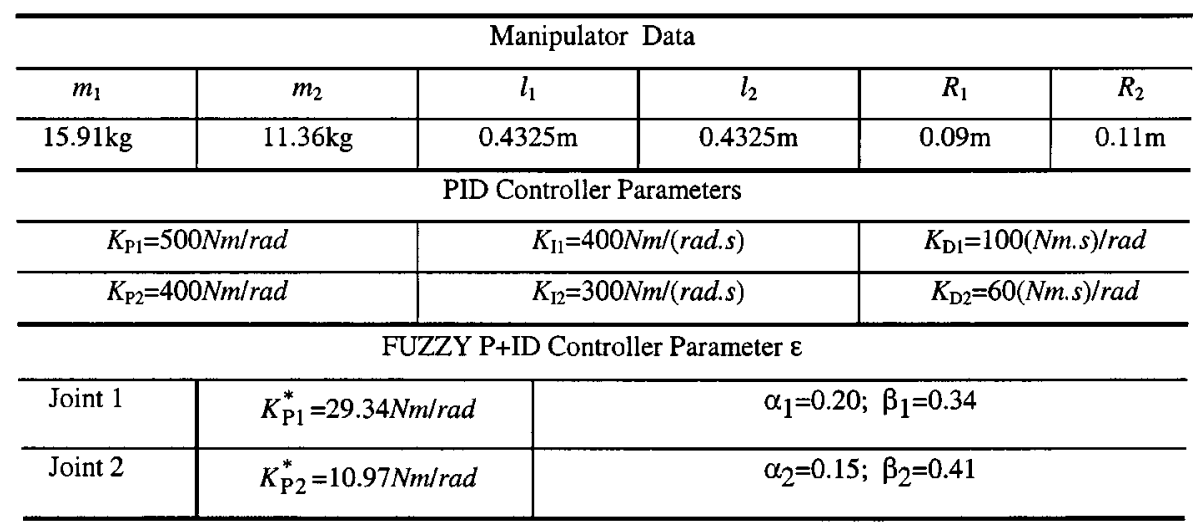

TABLE III

CONTROL PERFormance SPECIFICATIONS

\begin{tabular}{l|c|c|c|c|c|c|c}
\hline \multicolumn{2}{c|}{} & \multicolumn{2}{c|}{ Settling time } & \multicolumn{2}{c|}{ Overshoot } & \multicolumn{2}{c}{ steady-state error } \\
\hline \multicolumn{2}{c|}{} & Joint 1 & Joint 2 & Joint 1 & Joint 2 & Joint 1 & Joint 2 \\
\hline$\theta_{\text {ref1 }}=60^{\circ}$ & PID & $1.732 s$ & $2.334 s$ & $5.34^{\circ}$ & $5.46^{\circ}$ & $-0.05^{\circ}$ & $-0.187^{\circ}$ \\
\cline { 2 - 8 }$\theta_{\text {ref2 }}=50^{\circ}$ & FUZZY P+ID & $0.436 s$ & $0.474 s$ & $0.22^{\circ}$ & $0.006^{\circ}$ & $0.012^{\circ}$ & $0.0000^{\circ}$ \\
\hline$\theta_{\text {ref1 }}=60^{\circ}$ & PID (load change) & $0.462 s$ & $1.138 s$ & $0.778^{\circ}$ & $1.986^{\circ}$ & $-0.004^{\circ}$ & $-0.169^{\circ}$ \\
\cline { 2 - 8 }$\theta_{\text {ref2 } 2}=50^{\circ}$ & FUZZY P+ID (load change) & $0.442 s$ & $0.486 s$ & $0.114^{\circ}$ & $0.001^{\circ}$ & $0.002^{\circ}$ & $0.0015^{\circ}$ \\
\hline
\end{tabular}

and $K_{\mathrm{P} 2}^{*}$ of FUZZY $\mathrm{P}$ controllers are reduced, as listed in Table II. In the next step, the additional parameters ${ }^{1}\left(\alpha_{1}, \beta_{1}\right)$ and $\left(\alpha_{2}, \beta_{2}\right)$ of the FUZZY P+ID controllers are adjusted to improve control performance. As discussed above, increasing $\beta$ decreases the rise time. Based on experience, the ratio of $\beta$ and $\alpha$ should be $[1.5,3.0]$. Table II lists the parameters $\left(\alpha_{1}, \beta_{1}\right)$ and $\left(\alpha_{2}, \beta_{2}\right)$ used in the enhanced FUZZY P+ID controllers.

In the simulation studies, the sampling time $T$ has been chosen to be $2 \mathrm{~ms}$; the initial joint angles $\theta_{1}(0)$ and $\theta_{2}(0)$ were set to be $0^{\circ}$. We use the overshoot $M_{\mathrm{p}}$ (in this paper defined as difference between the desired and the maximum joint angle), the settling time $t_{\mathrm{s}}$ and steady-state error $e_{\mathrm{t} *}$ to quantify the control performance. Here, we choose step changes in configuration $\left(\theta_{\text {ref1 }}=60^{\circ}, \theta_{\text {ref2 }}=50^{\circ}\right)$ for the commanded trajectory. The solid lines of the top graphs in Fig. 6 show the dynamic responses of joints 1 and 2 obtained by the PID (left) and enhanced FUZZY P+ID (right) controllers. The bottom graphs in Fig. 6 show the applied torque at each motor joint. In the transient phase, PID control yields the overshoots for joints 1 and 2 of $M_{\mathrm{p}(1)}=$ $5.34^{\circ}$ and $M_{\mathrm{p}(2)}=5.46^{\circ}$ and the settling times $t_{\mathrm{s}(1)}=1.732 \mathrm{~s}$ and $t_{\mathrm{s}(2)}=2.334 \mathrm{~s}$. By enhanced FUZZY P+ID control, the overshoot is reduced to $M_{\mathrm{P}(1)}=0.22^{\circ}$ and $M_{\mathrm{P}(2)}=0.006^{\circ}$, and the settling times shorten to $t_{\mathrm{s}(1)}=0.436 \mathrm{~s}$ and $t_{\mathrm{s}(2)}=0.474 \mathrm{~s}$. In steady-state, PID control yields steady-state errors of $e_{\mathrm{t} *(1)}=-0.050^{\circ}$ and $e_{\mathrm{t} *(2)}=$ $-0.187^{\circ}$. The enhanced FUZZY P+ID control yields steady-state errors for joints 1 and 2 of $e_{\mathrm{t} *(1)}=0.012^{\circ}$ and $e_{\mathrm{t} *(2)}=0.000^{\circ}$. Fig. 6 shows that the Enhanced Fuzzy P+ID control achieves better performance (shorter settling time and less overshoot) with smaller peak applied torque.

In the next simulation, we investigated the effects of load changes on control performance. The forces and moment of the load are changed to be $f_{x}=25 \mathrm{~N}, f_{y}=30 \mathrm{~N}, n_{z}=20 \mathrm{Nm}$. The reference joint angles remain unchanged. The dotted curves in the top graphs of Fig. 6 show the time response to the step commands after the load changes. In this case, the change in overshoot from the unload to the load case is about $\Delta M_{\mathrm{p}(1)}=-5.12^{\circ}=$ and $\Delta M_{\mathrm{p}(2)}=-5.454^{\circ}$. The change in

${ }^{1}$ The subscripts denote the robot joint to which the parameters refer.
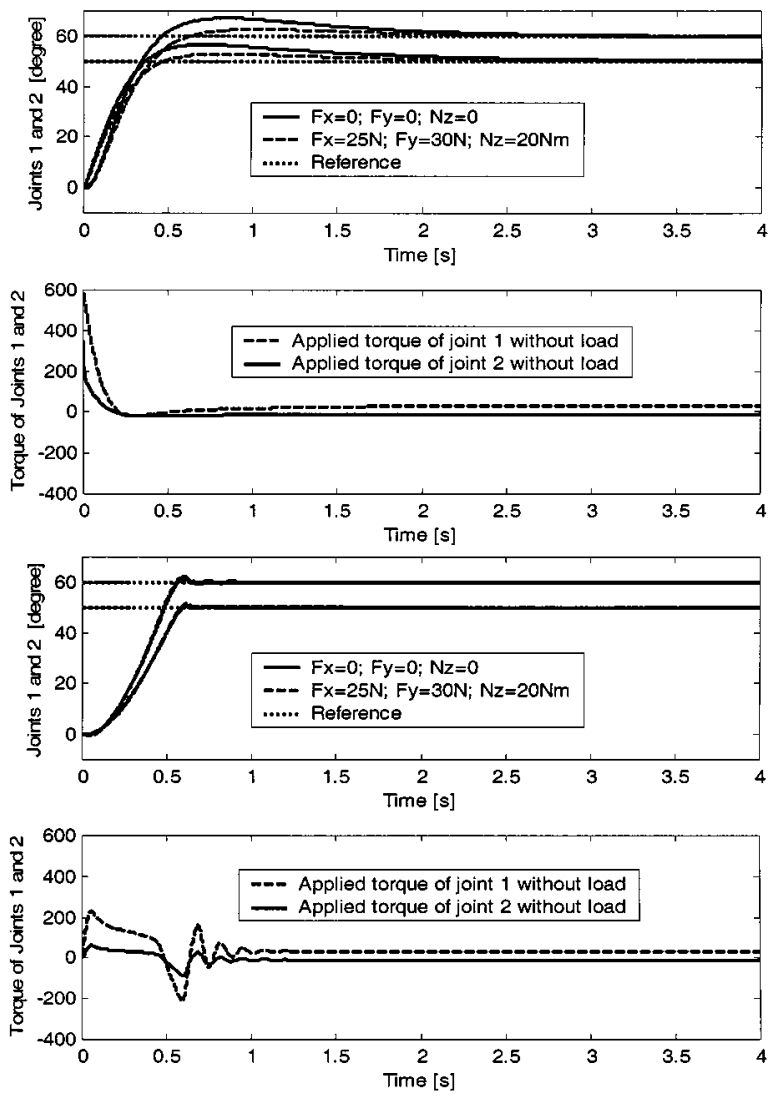

Fig. 6. Time responses of a manipulator in the presence of load changes. The top figures show the joint responses under PID (left) and enhanced FUZZY P+ID (right) control. The dashed, solid, and bold dashed lines represent the commanded response, the unloaded response, and the loaded response, respectively. The bottom plots show the applied torque signals for the PID (left) and enhanced FUZZY P+ID (right) control for the unloaded case. The dashed and solid lines correspond to the joint 1 and joint 2 torque, respectively. 

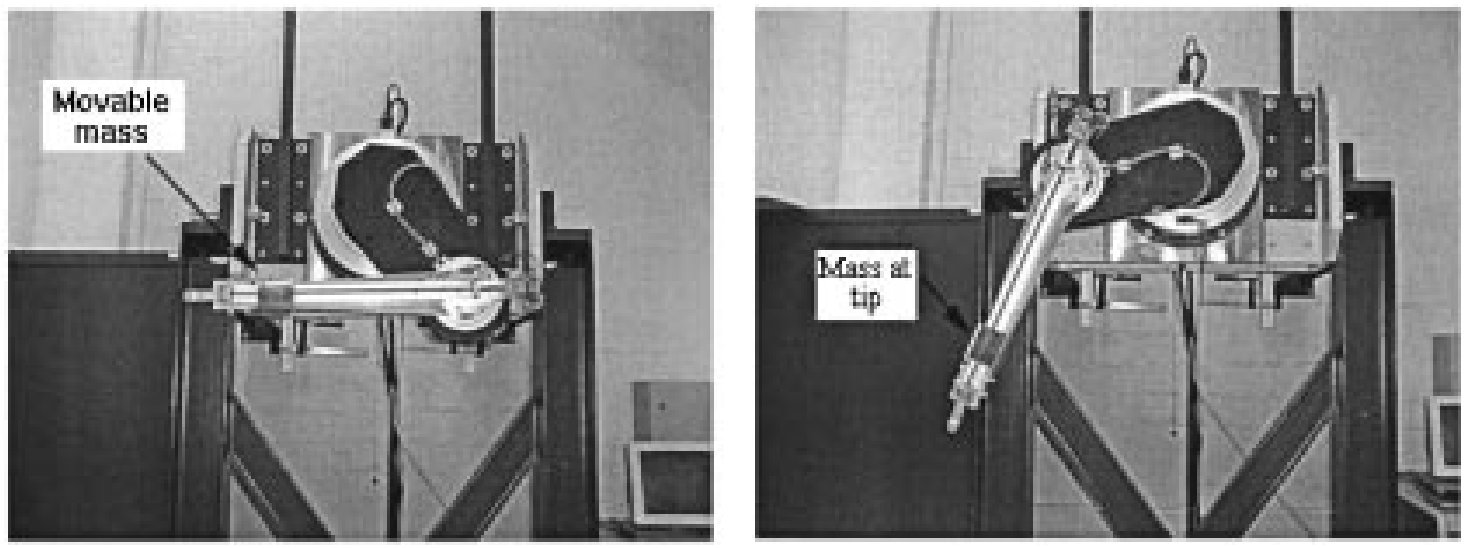

Fig. 7. Experimental direct drive two-link manipulator with a movable mass.
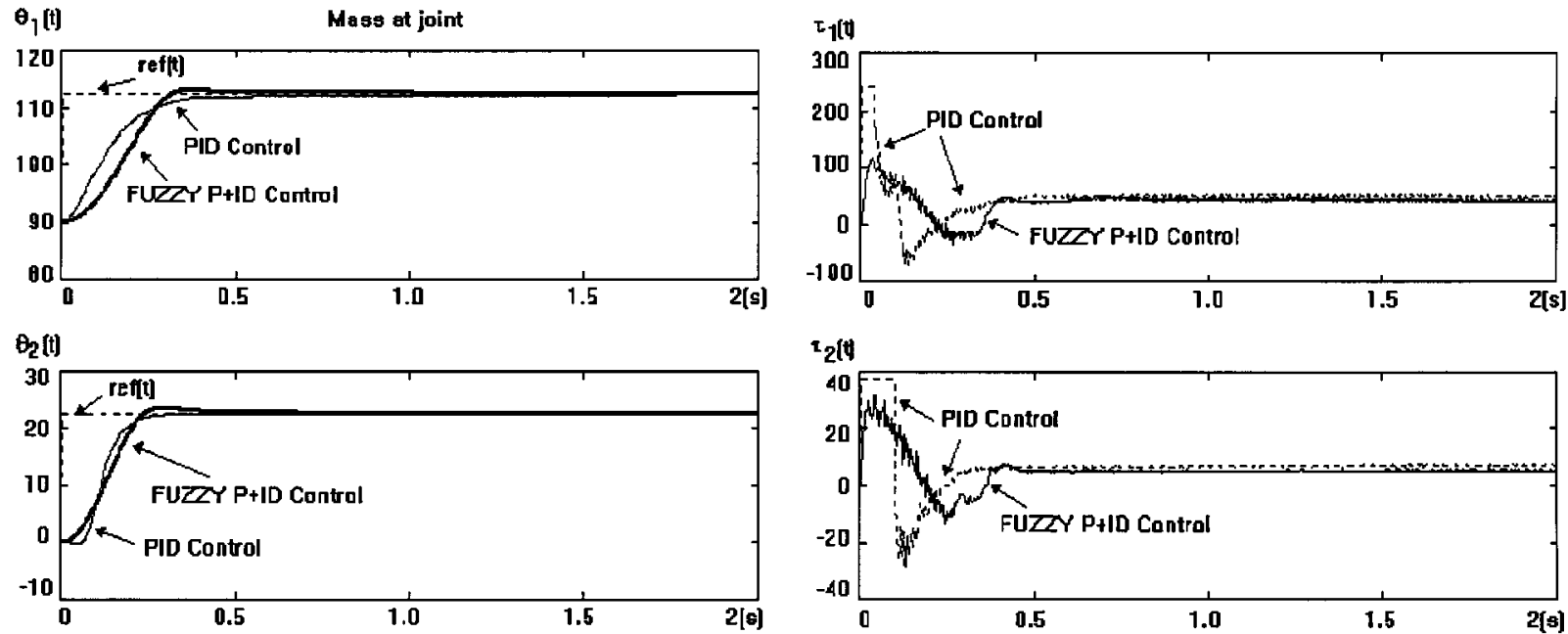

(a)
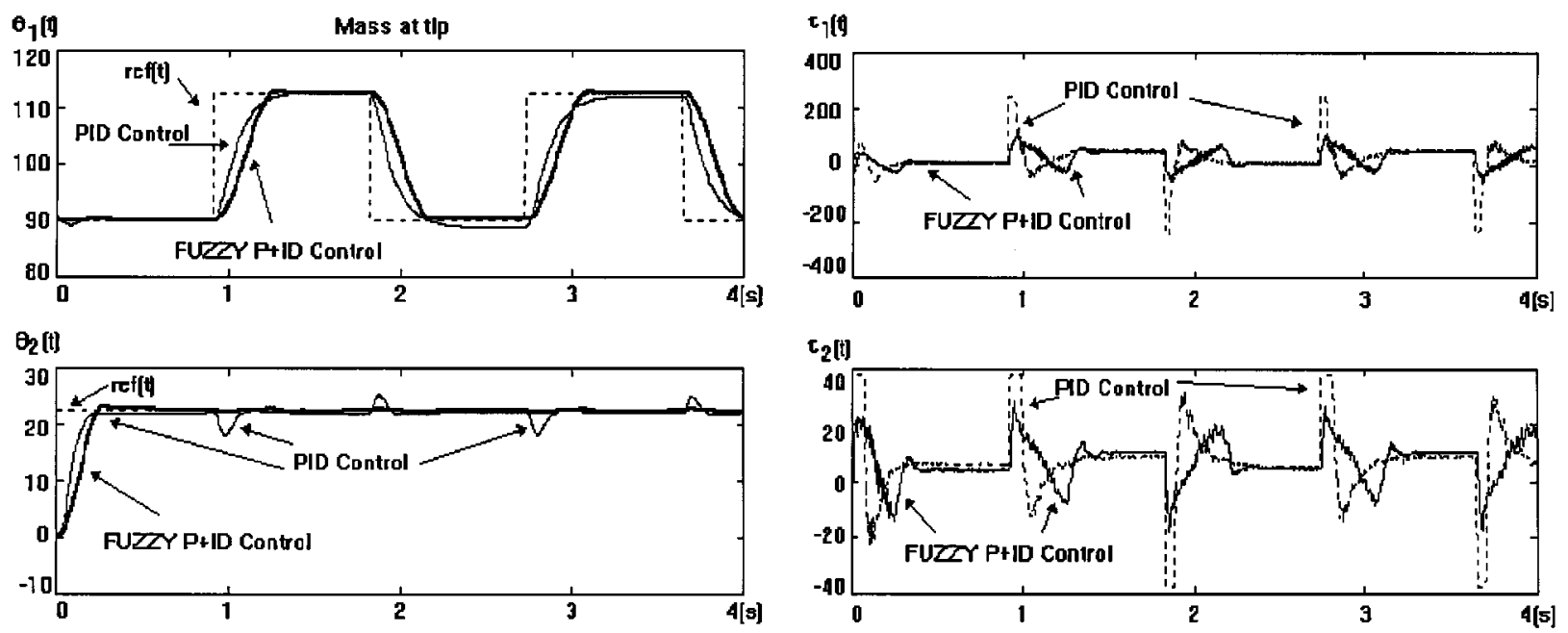

(c)

(d)

Fig. 8. Manipulator time responses for quad impulse and step control. (a) Joint time responses. (b) Applied torque to joints one and two. (c) Joint time responses. (d) Applied torque to joints one and two.

settling times from the unload to the load case is about $\Delta t_{\mathrm{s}(1)}=-1.27 \mathrm{~s}$ and $\Delta t_{\mathrm{s}(2)}=-1.196 \mathrm{~s}$. The change in steady-state errors $\Delta e_{\mathrm{t} *(1)}=-0.046^{\circ}$ and $\Delta e_{\mathrm{t} *(2)}=-0.018^{\circ}$. Using enhanced FUZZY P+ID control, all variations from the unload to the load case are much more smaller: $\Delta M_{\mathrm{p}(1)}=-0.106^{\circ}=$ and $\Delta M_{\mathrm{p}(2)}=0.005^{\circ}, \Delta t_{\mathrm{s}(1)}=-0.006 \mathrm{~s}$ and $\Delta t_{\mathrm{s}(2)}=-0.012 \mathrm{~s}$, $\Delta e_{\infty(1)}=0.01^{\circ}$ and $\Delta e_{\infty(2)}=-0.0015^{\circ}$. It is clear that the enhanced FUZZY P+ID control performance is much more robust to changes in the load than is PID control.

\section{EXPERIMENTS ON CONTROL OF A TWO-LINK MANIPULATOR}

Fig. 7 shows a direct drive two-link manipulator developed by the Institute for Robotics and Process Control, Technical University 
of Braunschweig, Germany. On the second link, a movable mass is mounted, which can be placed close to the second joint or near the tip of the link, hence it changes the inertial parameters of the manipulator. Thus, the manipulator is an ideal testbed to examine control performance in the presence of load uncertainty. The hardware system consists of a Window NT-based PC computer, the power stage of the motors, and an I/O-Card which is reading the encoder values and writing the new motor values. In the following experiments, the sample time $T$ of the control system is chosen to be $2 \mathrm{~ms}$, and both of the initial angles $\theta_{1}(0)$ and $\theta_{2}(0)$ are set to be $90^{\circ}$ and $0^{\circ}$ (i.e., both links in downward positions). First, the mass is located at the joint shown in Fig. 7. In this case, we use the approaches in [23] and [24] to tune the PID controllers to obtain an acceptable control performance. Actually, it is already very hard to further improve the control performance by tuning the PID controller parameters, because the applied joint torque already reaches its limit value. Based on the PID controller parameters, we adjust the additional parameters $\alpha$ and $\beta$ of the FUZZY P+ID controllers to improve control performance. The thin-solid curves in Fig. 8(a) show the step control responses achieved by the PID controllers. The thick-solid curves in Fig. 8(a) show the step time responses achieved by the FUZZY P+ID controllers. It is clear that control performance is improved after the PID controllers are replaced by the FUZZY P+ID ones. The solid and dotted curves in Fig. 8(b) plot the applied torque for joints 1 and 2 generated by the PID and FUZZY P+ID controllers, respectively. The maximum torque generated by PID control is much bigger than that required by the FUZZY P+ID control. Furthermore the applied joint torque required by PID control reaches its limit at the initial stage. This implies that the FUZZY P+ID controllers require less energy to control the manipulator.

In the next experiment, the adjustable mass is moved to the tip of the second link. The reference command for joint 1 is chosen as a quad impulse signal. The reference command for joint 2 remains unchanged. The joint responses and applied torques are shown in Fig. 8(c) and (d). The time response for joints one and two shown in Fig. 8(c), demonstrate two features of the FUZZY P+ID controllers that are superior to the PID controllers. First, notice the effect of gravity on the control performance. The quad impulse reference for joint 1 was specified so that joint 1 would change position a few times while joint 2 should be (nominally) stationary. The joint 1 command contains two step changes: one from $90^{\circ}$ to $112.47^{\circ}$; and the other from $112.47^{\circ}$ to $90^{\circ}$. Hence, the effect of gravity on dynamic responses is different for upward and downward motion. For the PID controllers, the tracking error, shown by the thin-solid curve in Fig. 8(c), from top to bottom is greater than the one from bottom to top due to the effect of gravity. However, the enhanced FUZZY P+ID controllers yield very small tracking errors in both cases, shown by the thick-solid curve in Fig. 8(c). These demonstrate that the FUZZY $\mathrm{P}+\mathrm{ID}$ controllers can effectively compensate gravity. Second, we discuss the coupling torque effects on control performance. In this experiment, the second joint first moves to its reference value; while the first joint remains stationary. By using PID control, the first joint deviates from its reference value because of the second joint motion. After the second joint reaches its reference value, the first joint is controlled to step between its reference values. In Fig. 8(c), it can be seen that the second link swings because it is coupled to the first link which is changing its joint position. By using enhanced FUZZY $\mathrm{P}+\mathrm{ID}$ controllers, however, the coupling torque effects are effectively compensated. This experiment demonstrates that the FUZZY P+ID controllers are superior with respect to performance robustness as compared to the PID controllers. The solid and dashed curves in Fig. 8(d) represent the torques computed by the FUZZY P+ID and PID controllers, respectively. It can be seen that the maximum torques of both joints using the FUZZY P+ID controller are significantly smaller than those of the PID controllers.

\section{CONCLUSION}

This paper presents an enhanced FUZZY P+ID controller with 25 rules. By using this controller, good performance in both transient and steady-state periods can be achieved. It is practical for improving the control performance of manipulators which already are controlled by PID type controllers. The structure of the FUZZY P+ID controller is very simple, since it is constructed by replacing the proportional term in the conventional PID controller with an incremental fuzzy logic controller. On the basis of the PID type controllers, only two additional parameters have to be adjusted to implement the FUZZY P+ID controller. These two parameters allow the controller to behave differently, depending on the values of $e$ and $\dot{e}$, without sacrificing the simplicity of the PID control structure. Thus, it is easy to achieve a desired control performance by tuning the FUZZY P+ID controller's parameter. In fact, the FUZZY P+ID parameters are not sensitive with respect to dynamic behavior of the system as compared with the PID parameters. The resulting FUZZY P+ID performance is less sensitive than PID control to changes in the dynamic model.

\section{APPENDIX}

This Appendix contains the FL rules when $\lambda=\beta$ and $\gamma=\alpha$

$$
\begin{aligned}
& \Delta u^{(1)}(k)=0 \quad e(k) \leq-\beta ; \beta \leq \dot{e}(k) . \\
& \Delta u^{(2,3)}(k)=\frac{\alpha(e(k)+\beta)}{(\beta-\alpha)} \\
& -\beta \leq e(k) \leq-\alpha ; \beta \leq \dot{e}(k) . \\
& \Delta u^{(4,5)}(k)=\beta+\frac{(\beta-\alpha) e(k)}{\beta} \\
& -\alpha \leq e(k) \leq 0 ; \beta \leq \dot{e}(k) . \\
& \Delta u^{(6,7,8,9,19)}(k)=\beta \quad 0 \leq e(k) ; \beta \leq \dot{e}(k) \text {. } \\
& \Delta u^{(11)}=\frac{\alpha(\dot{e}(k)-\beta)}{(\beta-\alpha)} \\
& e(k) \leq-\beta, \mathrm{b} \leq \dot{e}(k) \leq \beta . \\
& \Delta u^{(12)}= \begin{cases}-\frac{\alpha(\dot{e}(k)+e(k))}{(2 \dot{e}(k)-3 \beta+\alpha)}, & \text { if }|\mu(e . n b)| \leq|\mu(\dot{e} \cdot \mathrm{pb})| \\
\frac{\alpha(\dot{e}(k)+e(k))}{(2 e(k)+3 \beta-\alpha)}, & \text { if }|\mu(e . n b)|>|\mu(\dot{e} \cdot \mathrm{pb})|\end{cases} \\
& -\beta \leq e(k) \leq-\mathrm{b}, \mathrm{b} \leq \dot{e}(k) \leq \beta . \\
& \Delta u^{(13)}= \begin{cases}-\frac{\alpha(\dot{e}(k)+e(k))}{(2 e(k)+3 \beta-\alpha)}, & \text { if }|\mu(e . \mathrm{nb})| \leq|\mu(\dot{e} \cdot \mathrm{ps})| \\
-\frac{\alpha(\dot{e}(k)+e(k))}{(2 \dot{e}(k)-3 \beta+\alpha)}, & \text { if }|\mu(e . \mathrm{nb})|>|\mu(\dot{e} \cdot \mathrm{ps})|\end{cases} \\
& -\mathrm{b} \leq e(k) \leq-\alpha, \mathrm{b} \leq \dot{e}(k) \leq \beta . \\
& \Delta u_{f}^{(14)}=\left\{\begin{array}{c}
\frac{\alpha^{2} \dot{e}(k)-(\beta-\alpha)^{2} e(k)-\alpha \beta^{2}}{(2 \dot{e}(k)-3 \beta+\alpha)} \\
\text { if }|\mu(e . n s)| \leq|\mu(\dot{e} \cdot \mathrm{pb})| \\
\frac{2\left(\beta^{2}-\alpha^{2}\right) e(k)+\alpha^{2} \dot{e}(k)+\alpha \beta^{2}-2 \alpha^{3}}{(\beta-\alpha)(2 e(k)+3 \alpha)} \\
\text { if }|\mu(e . n s)| \leq|\mu(\dot{e} \cdot \mathrm{pb})|
\end{array}\right. \\
& -\alpha \leq e(k) \leq-\mathrm{a}, \mathrm{b} \leq \dot{e}(k) \leq \beta . \\
& \Delta u^{(15)}=\left\{\begin{array}{c}
\frac{\alpha(e(k)-\dot{e}(k))}{(2 e(k)-\alpha)} \\
\text { if }|\mu(e . \mathrm{ns})| \leq|\mu(\dot{e} \cdot \mathrm{ps})| \\
\frac{\alpha^{2} \dot{e}(k)-\left(\beta^{2}-\alpha^{2}\right) e(k)-\alpha \beta^{2}}{\alpha(2 \dot{e}(k)-3 \beta+\alpha)} \\
\text { if }|\mu(e . \mathrm{ns})| \leq|\mu(\dot{e} . \mathrm{ps})|
\end{array}\right. \\
& -\mathrm{a} \leq e(k) \leq 0, \mathrm{~b} \leq \dot{e}(k) \leq \beta \text {. }
\end{aligned}
$$




$$
\begin{aligned}
& \Delta u^{(16)}= \begin{cases}\frac{(\alpha+\beta) \dot{e}(k)-2 \beta^{2}}{(2 \dot{e}(k)-3 \beta+\alpha)}, & \text { if }|\mu(e . \mathrm{zo})| \leq|\mu(\dot{e} \cdot \mathrm{pb})| \\
\frac{\alpha \dot{e}(k)+2 \beta e(k)}{(2 e(k)+\alpha)}, & \text { if }|\mu(e . \mathrm{zo})|>|\mu(\dot{e} \cdot \mathrm{pb})|\end{cases} \\
& 0 \leq e(k) \leq \mathrm{a}, \mathrm{b} \leq \dot{e}(k) \leq \beta . \\
& \Delta u^{(17)}=\left\{\begin{array}{c}
\frac{(\alpha+\beta) e(k)-\alpha(2 \beta+\alpha)}{(2 e(k)-3 \alpha)} \\
\text { if }|\mu(e . z o)| \leq|\mu(\dot{e} . \mathrm{ps})| \\
\frac{(\alpha+\beta) \dot{e}(k)-2 \beta^{2}}{(2 \dot{e}(k)-3 \beta+\alpha)} \\
\text { if }|\mu(e . z o)|>|\mu(\dot{e} . \mathrm{ps})|
\end{array}\right. \\
& \mathrm{a} \leq e(k) \leq \alpha, \mathrm{b} \leq \dot{e}(k) \leq \beta . \\
& \Delta u^{(18,19,20)}(k)=\beta \quad \alpha \leq e(k) ; \beta \leq \dot{e}(k) . \\
& \Delta u^{(21)}=\frac{\alpha(\dot{e}(k)-\beta)}{(\beta-\alpha)} \\
& e(k) \leq-\beta, \alpha \leq \dot{e}(k) \leq \mathrm{b} . \\
& \Delta u^{(22)}= \begin{cases}\frac{\alpha(\dot{e}(k)+e(k))}{(2 \dot{e}(k)-3 \alpha+\beta)}, & \text { if }|\mu(e . \mathrm{nb})| \leq|\mu(\dot{e} . \mathrm{ps})| \\
\frac{\alpha(\dot{e}(k)+e(k))}{(2 e(k)+3 \beta-\alpha)}, & \text { if }|\mu(e . \mathrm{nb})|>|\mu(\dot{e} . \mathrm{ps})|\end{cases} \\
& -\beta \leq e(k) \leq-\mathrm{b}, \alpha \leq \dot{e}(k) \leq \mathrm{b} . \\
& \Delta u^{(23)}= \begin{cases}-\frac{\alpha(\dot{e}(k)+e(k))}{(2 e(k)+3 \alpha-\beta)}, & \text { if }|\mu(e . \mathrm{nb})| \leq|\mu(\dot{e} \cdot \mathrm{pb})| \\
\frac{\alpha(\dot{e}(k)+e(k))}{(2 \dot{e}(k)-3 \alpha+\beta)}, & \text { if }|\mu(e . \mathrm{nb})|>|\mu(\dot{e} \cdot \mathrm{pb})|\end{cases} \\
& -\mathrm{b} \leq e(k) \leq-\alpha, \alpha \leq \dot{e}(k) \leq \mathrm{b} . \\
& \Delta u^{(24)}=\left\{\begin{array}{l}
\frac{(\alpha+\beta) \dot{e}(k)+(\beta-\alpha) e(k)-2 \alpha^{2}}{(2 \dot{e}(k)-3 \alpha+\beta)} \\
\text { if }|\mu(e . \mathrm{ns})| \leq|\mu(\dot{e} \cdot \mathrm{ps})| \\
\frac{\left(\beta^{2}-\alpha^{2}\right) e(k)+\alpha^{2} \dot{e}(k)+\alpha \beta^{2}-2 \alpha^{3}}{(\beta-\alpha)(2 e(k)+3 \alpha)}
\end{array}\right. \\
& \text { if }|\mu(e . n s)|>|\mu(\dot{e} . \mathrm{ps})| \\
& -\alpha \leq e(k) \leq-\mathrm{a}, \alpha \leq \dot{e}(k) \leq \mathrm{b} . \\
& \Delta u^{(25)}=\left\{\begin{array}{l}
\frac{\alpha(e(k)-\dot{e}(k))}{(\alpha-2 e(k))} \\
\quad \text { if }|\mu(e . n s)| \leq|\mu(\dot{e} \cdot \mathrm{pb})| \\
\frac{(\beta+\alpha) \dot{e}(k)+(\beta-\alpha) e(k)-2 \alpha^{2}}{(2 \dot{e}(k)-3 \alpha+\beta)} \\
\text { if }|\mu(e . \mathrm{ns})|>|\mu(\dot{e} \cdot \mathrm{pb})|
\end{array}\right. \\
& -\mathrm{a} \leq e(k) \leq 0, \alpha \leq \dot{e}(k) \leq \mathrm{b} . \\
& \Delta u^{(26)}=\left\{\begin{array}{l}
\frac{2 \alpha \beta \dot{e}(k)+(\beta-\alpha)^{2} e(k)+\alpha^{2}(\beta+\alpha)}{\alpha(2 \dot{e}(k)-3 \beta+\alpha)} \\
\text { if }|\mu(e . z o)| \leq|\mu(\dot{e} . \mathrm{ps})| \\
\frac{\alpha(\dot{e}(k)+2 \beta e(k))}{(2 e(k)+\alpha)} \\
\text { if }|\mu(e . z o)|>|\mu(\dot{e} . \mathrm{ps})| \\
0 \leq e(k) \leq \mathrm{a}, \alpha \leq \dot{e}(k) \leq \mathrm{b} .
\end{array}\right. \\
& \Delta u^{(2 \tau)}=\left\{\begin{array}{l}
\frac{(\alpha+\beta) e(k)-\alpha(2 \beta+\alpha)}{(2 e(k)-3 \alpha)} \\
\text { if }|\mu(e . z o)| \leq|\mu(\dot{e} . \mathrm{pb})| \\
\frac{2 \alpha \beta \dot{e}(k)+(\beta-\alpha)^{2} e(k)-\alpha^{2}(\beta+\alpha)}{\alpha(2 \dot{e}(k)-3 \beta+\alpha)}
\end{array}\right. \\
& \text { if }|\mu(e . z o)|>|\mu(\dot{e} . \mathrm{pb})| \\
& 0 \leq e(k) \leq \mathrm{a}, \alpha \leq \dot{e}(k) \leq \mathrm{b} \text {. }
\end{aligned}
$$

$$
\begin{aligned}
\Delta u^{(28,29,30)}(k) & \\
= & \beta \quad \alpha \leq e(k) ; \alpha \leq \dot{e}(k) \leq \beta . \\
\Delta u^{(31)}=-\beta+\frac{(\beta-\alpha) \dot{e}(k)}{\alpha} & \quad(k) \leq-\beta, a \leq \dot{e}(k) \leq \alpha .
\end{aligned}
$$$$
\Delta u^{(32)}=\left\{\begin{array}{c}
-\frac{\alpha^{2} e(k)+\left(\beta^{2}-\alpha^{2}\right) \dot{e}(k)+\alpha\left(2 \alpha^{2}-\beta^{2}\right)}{(\beta-\alpha)(2 \dot{e}(k)-3 \alpha)} \\
\text { if }|\mu(e . \mathrm{nb})| \leq|\mu(\dot{e} \cdot \mathrm{ps})| \\
\frac{\alpha^{2} e(k)+(\beta-\alpha)^{2} \dot{e}(k)-\alpha \beta^{2}}{\alpha(2 e(k)+3 \beta-\alpha)} \\
\quad-\beta \leq e(k) \leq-\mathrm{b}, \mathrm{a} \leq \dot{e}(k) \leq \alpha .
\end{array}\right.
$$$$
\Delta u^{(33)}=\left\{\begin{array}{l}
-\frac{(\beta+\alpha) e(k)+(\beta-\alpha) \dot{e}(k)+2 \alpha^{2}}{(\beta-\alpha)(2 \dot{e}(k)-3 \alpha)} \\
\quad \text { if }|\mu(e . n b)| \leq|\mu(\dot{e} . z o)| \\
-\frac{\alpha^{2} e(k)+\left(\beta^{2}-\alpha^{2}\right) \dot{e}(k)+\alpha\left(2 \alpha^{2}-\beta^{2}\right)}{(\beta-\alpha)(2 \dot{e}(k)-3 \alpha)} \\
\quad \text { if }|\mu(e . n b)|>|\mu(\dot{e} . z o)| \\
-\mathrm{b} \leq e(k) \leq-\alpha, a \leq \dot{e}(k) \leq \alpha .
\end{array}\right.
$$$$
-\mathrm{b} \leq e(k) \leq-\alpha, \mathrm{a} \leq \dot{e}(k) \leq \alpha .
$$$$
\Delta u^{(34)}= \begin{cases}\frac{\alpha(\dot{e}(k)+e(k))}{(3 \alpha-2 \dot{e}(k))}, & \text { if }|\mu(e . \mathrm{ns})| \leq|\mu(\dot{e} . \mathrm{ps})| \\ \frac{\alpha(\dot{e}(k)+e(k))}{(2 e(k)+3 \alpha)}, & \text { if }|\mu(e . \mathrm{ns})|>|\mu(\dot{e} . \mathrm{ps})|\end{cases}
$$$$
-\alpha \leq e(k) \leq-\mathrm{a}, \alpha \leq \dot{e}(k) \leq \mathrm{b} .
$$$$
\Delta u^{(35)}= \begin{cases}\frac{\alpha(\dot{e}(k)+e(k))}{(\alpha-2 \dot{e}(k))}, & \text { if }|\mu(e . \mathrm{ns})| \leq|\mu(\dot{e} . \mathrm{zo})| \\ \frac{\alpha(\dot{e}(k)+e(k))}{(3 \alpha-2 e(k))}, & \text { if }|\mu(e . \mathrm{ns})|>|\mu(\dot{e} . \mathrm{zo})|\end{cases}
$$$$
-\mathrm{a} \leq e(k) \leq 0, \alpha \leq \dot{e}(k) \leq \mathrm{b} .
$$

$$
\Delta u^{(36)}=\left\{\begin{array}{c}
\frac{(\beta-\alpha) e(k)-\alpha \dot{e}(k)+2 \alpha^{2}}{(2 \dot{e}(k)-3 \alpha)} \\
\text { if }|\mu(e . \mathrm{zo})| \leq|\mu(\dot{e} \cdot \mathrm{ps})| \\
\frac{(\beta+\alpha) e(k)+\alpha \dot{e}(k)}{(2 e(k)+3 \alpha)} \\
\text { if }|\mu(e . \mathrm{zo})|>|\mu(\dot{e} \cdot \mathrm{ps})| \\
0 \leq e(k) \leq \mathrm{a}, \alpha \leq \dot{e}(k) \leq \mathrm{b} .
\end{array}\right.
$$

$$
\Delta u^{(37)}=\left\{\begin{array}{c}
\frac{(\beta-\alpha) e(k)-\alpha \dot{e}(k)+2 \alpha^{2}}{(3 \alpha-2 e(k))} \\
\text { if }|\mu(e . z o)| \leq|\mu(\dot{e} . z o)| \\
\frac{(\beta-\alpha) e(k)-\alpha \dot{e}(k)+2 \alpha^{2}}{(3 \alpha-2 \dot{e}(k))} \\
\text { if }|\mu(e . z o)|>|\mu(\dot{e} . z o)|
\end{array}\right.
$$$$
\mathrm{a} \leq e(k) \leq \alpha, \alpha \leq \dot{e}(k) \leq \mathrm{b} .
$$

$$
\begin{aligned}
& \Delta u^{(38)}=\left\{\begin{array}{l}
\frac{(\beta+\alpha) \dot{e}(k)-\alpha(\alpha+2 \beta)}{(2 \dot{e}(k)-3 \alpha)} \\
\text { if }|\mu(e . \mathrm{ps})| \leq|\mu(\dot{e} . \mathrm{ps})| \\
\frac{\left(\beta^{2}-\alpha^{2}\right) \dot{e}(k)+2 \alpha \beta e(k)-\alpha^{2}(\alpha+\beta)}{\alpha(2 e(k)-3 \alpha+\beta)} \\
\quad \text { if }|\mu(e . \mathrm{ps})|>|\mu(\dot{e} . \mathrm{ps})|
\end{array}\right. \\
& \alpha \leq e(k) \leq \mathrm{b}, \alpha \leq \dot{e}(k) \leq \mathrm{b} .
\end{aligned}
$$




$$
\begin{aligned}
& \Delta u^{(39)}=\left\{\begin{array}{c}
\frac{(\beta+\alpha) e(k)-2 \beta^{2}}{(2 e(k)-3 \beta+\alpha)} \\
\text { if }|\mu(e . p s)| \leq|\mu(\dot{e} . z o)| \\
\frac{(\beta+\alpha) \dot{e}(k)-\alpha(\alpha+2 \beta)}{(2 \dot{e}(k)-3 \alpha)} \\
\text { if }|\mu(e . p s)|>|\mu(\dot{e} . z o)|
\end{array}\right. \\
& \mathrm{b} \leq e(k) \leq \beta, \alpha \leq \dot{e}(k) \leq \mathrm{b} . \\
& \Delta u^{(40)}(k)=\beta \quad \beta \leq e(k) ; \mathrm{a} \leq \dot{e}(k) \leq \alpha . \\
& \Delta u^{(41)}=-\beta+\frac{(\beta-\alpha) \dot{e}(k)}{\alpha} \\
& e(k) \leq-\beta, 0 \leq \dot{e}(k) \leq \mathrm{a} . \\
& \Delta u^{(42)}=\left\{\begin{array}{l}
\frac{\alpha(e(k)-\dot{e}(k))}{(2 \dot{e}(k)+\alpha)} \\
\text { if }|\mu(e . n s)| \leq|\mu(\dot{e} . z o)| \\
\frac{(\beta-\alpha)^{2} \dot{e}(k)-\alpha^{2} e(k)-\alpha^{2} \beta}{\alpha(2 e(k)+3 \beta-\alpha)} \\
\text { if }|\mu(e . n s)|>|\mu(\dot{e} . z o)|
\end{array}\right. \\
& -\beta \leq e(k) \leq-\mathrm{b}, 0 \leq \dot{e}(k) \leq \mathrm{a} \text {. } \\
& \Delta u^{(43)}=\left\{\begin{array}{l}
-\frac{(\beta-\alpha) \dot{e}(k)+(\beta+\alpha)(k)+2 \alpha^{2}}{(2 e(k)+3 \alpha-\beta)} \\
\quad \text { if }|\mu(e . n b)| \leq|\mu(\dot{e} . z o)| \\
-\frac{\alpha(e(k)-\dot{e}(k))}{(2 \dot{e}(k)+\alpha)} \\
\quad \text { if }|\mu(e . n b)|>|\mu(\dot{e} . z o)| \\
-\mathrm{b} \leq e(k) \leq-\alpha, 0 \leq \dot{e}(k) \leq \mathrm{a} .
\end{array}\right. \\
& \Delta u^{(44)}= \begin{cases}+\frac{\alpha(\dot{e}(k)+e(k))}{(2 \dot{e}(k)+\alpha)}, & \text { if }|\mu(e . n s)| \leq|\mu(\dot{e} . z o)| \\
+\frac{\alpha(\dot{e}(k)+e(k))}{(2 e(k)-3 \alpha)}, & \text { if }|\mu(e . n s)|>|\mu(\dot{e} . z o)|\end{cases} \\
& -\alpha \leq e(k) \leq-\mathrm{a}, 0 \leq \dot{e}(k) \leq \mathrm{a} . \\
& \Delta u^{(45)}= \begin{cases}+\frac{\alpha(\dot{e}(k)+e(k))}{(\alpha-2 e(k))}, & \text { if }|\mu(e . \mathrm{ns})| \leq|\mu(\dot{e} \cdot \mathrm{ps})| \\
-\frac{\alpha(\dot{e}(k)+e(k))}{(2 \dot{e}(k)+\alpha)}, & \text { if }|\mu(e . \mathrm{ns})|>|\mu(\dot{e} . \mathrm{ps})|\end{cases} \\
& -\mathrm{a} \leq e(k) \leq 0,0 \leq \dot{e}(k) \leq \mathrm{a} . \\
& \Delta u^{(46)}=\left\{\begin{array}{c}
\frac{(\beta+\alpha) \dot{e}(k)+\alpha e(k)}{(2 \dot{e}(k)+\alpha)} \\
\text { if }|\mu(e . z o)| \leq|\mu(\dot{e} . z o)| \\
\frac{(\beta+\alpha) e(k)+\alpha \dot{e}(k)}{(2 e(k)+\alpha)} \\
\text { if }|\mu(e . z o)|>|\mu(\dot{e} . z o)|
\end{array}\right. \\
& 0 \leq e(k) \leq \mathrm{a}, 0 \leq \dot{e}(k) \leq \mathrm{a} . \\
& \Delta u^{(47)}=\left\{\begin{array}{c}
\frac{(\beta-\alpha) \dot{e}(k)-\alpha e(k)+2 \alpha^{2}}{(3 \alpha-2 e(k))} \\
\text { if }|\mu(e . z o)| \leq|\mu(\dot{e} . \mathrm{ps})| \\
\frac{(\beta+\alpha) \dot{e}(k)+\alpha e(k)}{(2 \dot{e}(k)+\alpha)} \\
\text { if }|\mu(e . z o)|>|\mu(\dot{e} . \mathrm{ps})|
\end{array}\right. \\
& \mathrm{a} \leq e(k) \leq \alpha, 0 \leq \dot{e}(k) \leq \mathrm{a} . \\
& \Delta u^{(48)}=\left\{\begin{array}{l}
\frac{2 \beta \dot{e}(k)+\alpha e(k)}{(2 \dot{e}(k)+\alpha)} \\
\text { if }|\mu(e . p s)| \leq|\mu(\dot{e} . z o)| \\
\frac{(\beta-\alpha)^{2} \dot{e}(k)+2 \alpha \beta e(k)-\alpha^{2}(\beta+\alpha)}{\alpha(2 e(k)-3 \alpha+\beta)} \\
\text { if }|\mu(e . p s)|>|\mu(\dot{e} . z o)|
\end{array}\right.
\end{aligned}
$$

$$
\begin{gathered}
\alpha \leq e(k) \leq \mathrm{b}, 0 \leq \dot{e}(k) \leq \mathrm{a} . \\
\Delta u^{(49)}=\left\{\begin{array}{l}
\frac{(\beta+\alpha) e(k)-2 \beta^{2}}{(2 e(k)-3 \beta+\alpha)}, \quad \text { if }|\mu(e . p s)| \leq|\mu(\dot{e} . \mathrm{ps})| \\
\frac{2 \beta \dot{e}(k)+\alpha e(k)}{(2 \dot{e}(k)+\alpha)}, \quad \text { if }|\mu(e . p s)|>|\mu(\dot{e} . \mathrm{ps})|
\end{array}\right. \\
\Delta u^{(50)}(k)=\beta \quad \beta \leq e(k) \leq \beta, 0 \leq \dot{e}(k) \leq \mathrm{a} .
\end{gathered}
$$

\section{REFERENCES}

[1] L. A. Zadeh, "Fuzzy sets," Inf. Contr., vol. 8, pp. 339-353, 1965.

[2] H. R. Berenji, "Fuzzy logic controllers," in An Introduction to Fuzzy Logic Application in Intelligent Systems, R. R. Yager and L. A. Zadeh, Eds. Boston, MA: Kluwer, 1992.

[3] H.-J. Zimmermann, Fuzzy Sets Theory and Its Applications. Norwell, MA: Kluwer-Nijhoff, 1984.

[4] E. H. Mamdani, "Application of fuzzy algorithm for control of simple dynamic plant," Proc. Inst. Elect. Eng., vol. 121, pp. 1585-1588, 1974.

[5] S. Tzafestas and N. Papanikolopoulos, "Incremental fuzzy expert PID control," IEEE Trans. Ind. Electron., vol. 37, pp. 365-371, Oct. 1990.

[6] W. Li and B. Zhang, "Fuzzy control of robotic manipulators in the presence of joint friction and loads changes," in Proc. ASME Int. Comput. Eng. Conf., 1993.

[7] H. R. Berenji and P. Khedkar, "Learning and tuning fuzzy logic controllers through reinforcements," IEEE Trans. Neural Networks, vol. 3, pp. 724-740, Sept. 1992.

[8] J. S. R. Jang, "Self-learning fuzzy controllers based on temporal back propagation," IEEE Trans. Neural Networks, vol. 3, pp. 714-723, 1992.

[9] T. J. Procyk and E. H. Mamdani, "A linguistic self-organizing process controller," Automatica, vol. 15, pp. 15-30, Sept. 1979.

[10] E. M. Scharf and N. J. Mandic, "The application of a fuzzy controller to the control of a multi-degree-freedom robot arm," Ind. Applicat. Fuzzy Control, pp. 41-61, 1985.

[11] W. Li, "Optimization of a fuzzy logic controller using neural network," in Proc. IEEE World Congr. Computat. Intell., vol. 1, 1994, pp. 223-227.

[12] F. Chen and H. Khalil, "Adaptive control of a class of a nonlinear discrete-time systems using neural networks," IEEE Trans. Automat. Contr., vol. 40, pp. 791-801, May 1993.

[13] S. Commuri and F. Lewis, "CMAC neural networks for control of nonlinear dynamical systems: Structure, stability, and passivity," in Proc. IEEE Int. Symp. Intell. Control, Aug. 1995.

[14] J. A. Farrell, "Stability and approximator convergence in nonparametric nonlinear adaptive control," IEEE Trans. Neural Networks, vol. 9, pp. 1008-1020, Sept. 1998.

[15] F. Lewis, A. Yesildirek, and K. Liu, "Multilayer neural-net robot controller with guaranteed tracking performance," IEEE Trans. Neural Networks, vol. 7, pp. 388-399, Mar. 1996.

[16] M. Polycarpou, "Stable adaptive neural control scheme for nonlinear systems," IEEE Trans. Automat. Contr., vol. 41, pp. 447-451, Mar. 1996.

[17] R. Sanner and J. Slotine, "Gaussian networks for direct adaptive control,” IEEE Trans. Neural Networks, vol. 3, pp. 837-863, Nov. 1992.

[18] H. Ying, W. Siler, and J. J. Buckeley, "Fuzzy control theory: A nonlinear case," Automatica, vol. 26, pp. 513-520, 1990.

[19] H. A. Malki, H. D. Li, and G. Chen, "New design and stability analysis of fuzzy proportional-derivative control systems," IEEE Trans. Fuzzy Syst., vol. 2, pp. 245-254, Aug. 1994.

[20] D. Misir, H. A. Malki, and G. Chen, "Design and analysis of fuzzy proportional-integral-derivative controller," Fuzzy Sets Syst., vol. 79, pp. 297-314, 1996.

[21] W. Li, "Design of a hybrid fuzzy logic proportional plus conventional integral-derivative controller," IEEE Trans. Fuzzy Syst., vol. 6, pp. 449-463, Aug. 1998.

[22] X. G. Chang, "Design of hybrid fuzzy controllers and modeling of complicated systems under uncertainty by soft computing," $\mathrm{Ph}$.D. dissertation, Dept. Comput. Sci. Technol., Tsinghua University, Beijing, China, 2000.

[23] C. A. Desoer and M. Vidyasagar, Feedback System: Input-Output Properties. New York: Academic, 1975.

[24] R. J. P. de Figuerredo and G. Chen, Nonlinear Feedback Control System: An Operator Theory Approach. New York: Academic, 1993.

[25] M. Brady et al., Robot Motion: Planning and Control. Cambridge, MA: MIT Press, 1982.

[26] J. J. Craig, Introduction to Robotics Mechanics and Control. MA: Addison-Wesley, 1986. 